\title{
Conductive Hearing Loss Produces a Reversible Binaural Hearing Impairment
}

\author{
David R. Moore, Jemma E. Hine, Ze Dong Jiang, Hiroaki Matsuda, Carl H. Parsons, and Andrew J. King \\ University Laboratory of Physiology, Oxford OX1 3PT, United Kingdom
}

Conductive hearing loss, produced by otitis media with effusion, is widespread in young children. However, little is known about its short- or long-term effects on hearing or the brain. To study the consequences of a conductive loss for the perception and processing of sounds, we plugged the left ear canal of ferrets for 7-15 months during either infancy or adulthood. Before or during plugging, the ferrets were trained to perform a binaural task requiring the detection of a $500 \mathrm{~Hz}$ tone, positioned $90^{\circ}$ to the right, that was masked by two sources of broad-band noise. In one condition ("control"), both noise sources were $90^{\circ}$ right and, in the second condition ("bilateral"), one noise source was moved to $90^{\circ}$ left. Normal ferrets showed binaural unmasking: tone detection thresholds were lower (mean $10.1 \mathrm{~dB}$ ) for the bilateral condition than for the control condition. Both groups of ear-plugged ferrets had reduced unmasking; the mean residual unmasking was $2.3 \mathrm{~dB}$ for the infant and $0.7 \mathrm{~dB}$ for the adult ear-plugged animals. After unplugging, unmasking increased in both groups (infant, 7.1 $\mathrm{dB}$; adult, $6.9 \mathrm{~dB}$ ) but not to normal levels. Repeated testing during the 22 months after unplugging revealed a gradual return to normal levels of unmasking. These results show that a unilateral conductive hearing loss, in either infancy or adulthood, impairs binaural hearing both during and after the hearing loss. They show scant evidence for adaptation to the plug and demonstrate a recovery from the impairment that occurs over a period of several months after restoration of normal peripheral function.

Key words: deafness; auditory plasticity; otitis media; binaural unmasking; ferret; ear plug
Almost everyone experiences a conductive hearing loss, a reduction in sound transmission to the inner ear, at some time. Common causes of conductive losses include otitis media with eff usion (OME; "glue ear"), excess cerumen in the ear canal, and otosclerosis (stapes fixation). OME, fluid in the middle ear, is especially common in infancy (Hogan et al., 1997). Although it has been associated with language, learning, and social difficulties in later life (Haggard and Hughes, 1993; Bluestone and Klein, 1995), the immediate and long-term consequences of OME remain obscure. Psychoacoustic studies of children with a history of OME have shown impairments in binaural processing, both during and after the OME (Moore et al., 1991; Pillsbury et al., 1991). Otosclerosis, which generally occurs in adulthood (Lucente and Sobol, 1988), also impairs binaural hearing, before and after surgical correction (Hall et al., 1990).

The perceptual effects of conductive hearing loss that outlive the peripheral impairment have been attributed to neural plasticity in the central auditory system. This view is supported by analogy with the effects of visual deprivation on the striate cortex (Wiesel, 1982), and by studies relating conductive hearing loss to anatomical (Feng and Rogowski, 1980; Smith et al., 1983; Moore

\footnotetext{
Received June 3, 1999; revised July 20, 1999; accepted July 21, 1999.

This research was supported by grants from the Medical Research Council, the Wellcome Trust, and Defeating Deafness (the Hearing Research Trust). We are indebted to Russell Martin, Sue Spires, and Gao Xia for help in training and testing the animals, and to Duncan Fleming and his staff for their superb care of the animals.

Correspondence should be addressed to David R. Moore, University Laboratory of Physiology, Parks Road, Oxford OX1 3PT, UK.

Dr. Hine's present address: Medical Research Council Institute of Hearing Research, Royal South Hants Hospital, Southampton SO14 0YG, UK.

Dr. Jiang's present address: Department of Paediatrics, University of Oxford, John Radcliffe Hospital, Oxford OX3 9DU, UK.

Copyright (C) 1999 Society for Neuroscience $\quad 0270-6474 / 99 / 198704-08 \$ 05.00 / 0$
}

et al., 1989) and physiological (Silverman and Clopton, 1977; Moore and Irvine, 1981; Knudsen, 1985; King et al., 1988; Mogdans and Knudsen, 1994) changes in the brainstem. Behavioral studies of experimentally induced conductive hearing loss in adult humans have been limited to relatively short-term plugging of the ear canal (Florentine, 1976; Moore, 1993; Slattery and Middlebrooks, 1994; McPartland et al., 1997). Wilmington and colleagues (1994) found that humans born with a unilateral ear canal atresia generally performed poorly on various binaural tasks. After restorative surgery, performance on most tasks rapidly improved to normal levels. However, they found that freefield sound localization was altered more by the conductive hearing loss than were tasks based purely on interaural comparisons. In barn owls, monaural occlusion during infancy leads to adaptive shifts in sound localization (Knudsen et al., 1984a). (Variants on the term "adaptation" are used throughout this paper to denote changes in auditory function produced by changes in auditory experience. The term is not intended to imply a beneficial effect or to invoke a particular neural mechanism.) If the ear is unplugged before sexual maturation, normal localization can be regained (Knudsen et al., 1984b). Older birds cannot adapt, either to an ear plug or to removal of a plug placed earlier in life.

These studies raise questions about the task and age dependency of impairments produced by altered auditory inputs. In this paper, we examine the effect of long-term, unilateral ear plugging in infant and adult ferrets on free-field binaural unmasking (BU) (Hine et al., 1994). BU was chosen because it is the test most commonly examined in the cited human studies, and because it is closely related to abilities of great importance to humans: the perceptual detection, identification, and discrimination of sounds in noisy environments (Moore, 1997). 


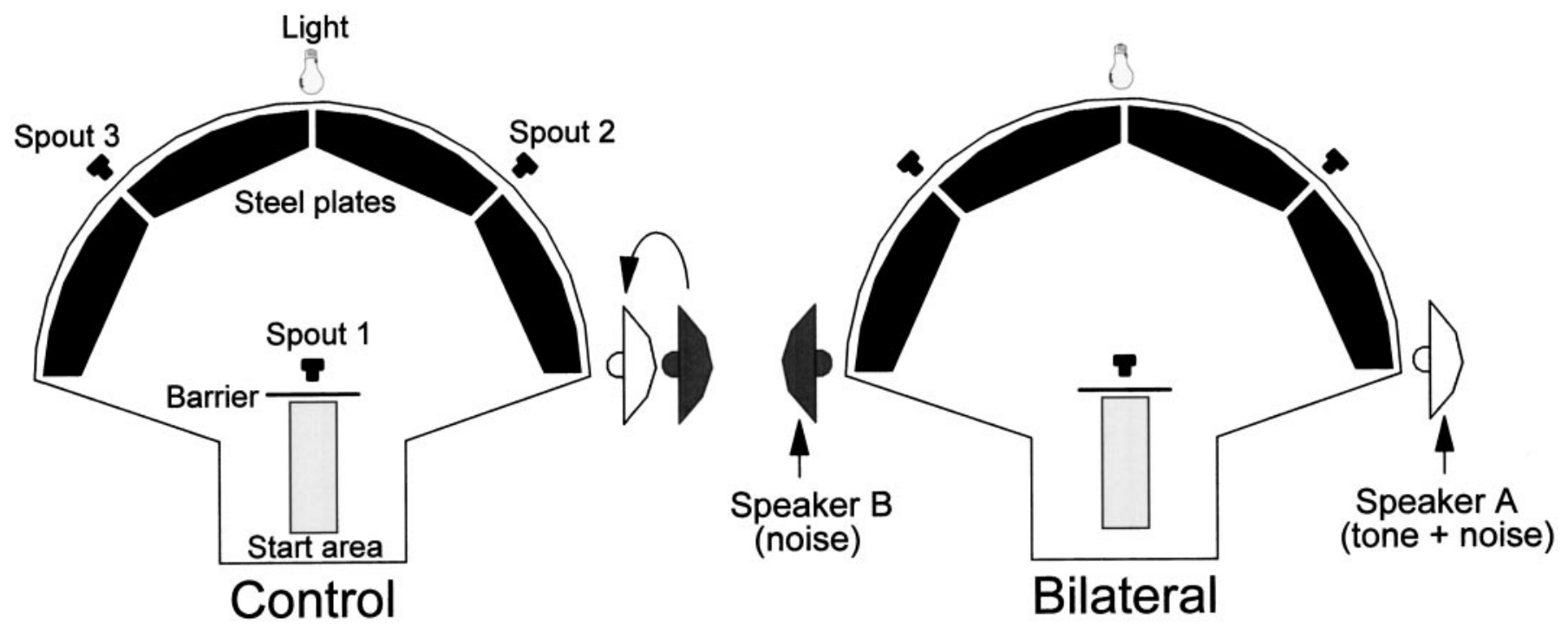

Figure 1. Configuration of apparatus in each condition. A top-down schematic of the test chamber showing the different positions of speakers used in the Control (noise and $500 \mathrm{~Hz}$ tone $90^{\circ}$ right) and Bilateral (tone $90^{\circ}$ right, noise $90^{\circ}$ right and left) conditions. In the Control condition, Speaker B was actually stacked vertically on top of Speaker $A$, as indicated by the arrow. On each trial, ferrets entered the start area and licked a water delivery spout (Spout 1) before proceeding to Spouts 2 and 3. Spout 1 to speaker distance was $0.65 \mathrm{~m}$.

The behavioral methods used in this study have been published previously (Hine et al., 1994).

\section{MATERIALS AND METHODS}

Animals. Sixteen pigmented ferrets (Mustela putorius furo), born from timed pregnancies and raised in this Department's animal care facility, were trained and tested. Data for normal, adult animals $(n=4)$ were obtained from a previous study (Hine et al., 1994). These ferrets had clean ears, peaked tympanograms (see below), and behavioral thresholds $[$ mean $=17.5 \mathrm{~dB}$ sound pressure level $(\mathrm{SPL}), \mathrm{SEM}=1.7$ at $500 \mathrm{~Hz}$ ] within the normal range for ferrets tested in our facility. They were at least 3 months of age when training began and 6-24 months at the time of testing for BU. Infant-plug ferrets $(n=8)$ had a plug inserted in the left ear [one ferret (9405) was reared and tested with a plug in the right ear] between postnatal day (P) 25 and P29. Hearing in the ferret begins between P26 and P30 (Moore and Hine, 1992). The ear was plugged continuously for a further 7-15 months. Training began from 3 months of age. Testing began from 6 months and continued for up to 22 months after removal of the plug. Adult-plug ferrets $(n=4)$ had a plug inserted in the left ear from after 5 months of age. The ear was continuously plugged for a further 2-8 months. Training began either before (F9121, F9124), or 2 months after (F9549, F9550) insertion of the plug. Testing began either immediately (F9121, F9124) or 6 months after (F9549, F9550) plugging. Testing continued for 2 months with the plug inserted and for up to 14 months after removal of the plug.

During training and testing the ferrets received vitamin-supplemented water reinforcement and were deprived of water in their home cages. They were maintained on a diet of high-protein dry pellets, to which they had access ad libitum. After a series of training or testing sessions, which lasted a maximum of $12 \mathrm{~d}$, the ferrets were allowed unlimited home cage access to water and wet food for at least a further $2 \mathrm{~d}$. The ferrets were weighed weekly, and their general health was monitored closely.

Earplugs. Earplugs were inserted, inspected, and removed under sedation with Domitor (medetomidine hydrochloride, $0.05-0.15 \mathrm{ml} / \mathrm{kg}$, i.m.). The earplug consisted of a pellet $\left(\sim 30 \mathrm{~mm}^{3}\right)$, cut from an E.A.R. Classic human foam earplug, which was compressed and inserted into the left ear canal. In addition, a silicone polymer hearing aid impression compound (Dreve Otoform-K) was mixed and molded into the concha of the outer ear. The margins of the silicone plug were secured to the pinna with Vetbond tissue adhesive. Plugs in all animals were checked at least once a day and replaced as necessary.

The sound attenuation produced by the earplugs was checked using auditory brainstem response (ABR) audiometry. Normally hearing adult ferrets $(n=3)$ were anesthetized with Sagatal (sodium pentobarbital; 45 $\mathrm{mg} / \mathrm{kg}$, i.p.). One inner ear was exposed and destroyed surgically (Moore and Kowalchuk, 1988). Differential recordings of the ABR were obtained from needle electrodes inserted into the scalp over the midline of the head and the bulla of the intact ear, as detailed previously (Morey and Carlile, 1990; Moore and Hine, 1992). Briefly, the ferret was placed in a sound-insulated chamber lined on the walls and ceiling with acoustically absorbent foam. Transient tone $(1,2,4,8$, and $16 \mathrm{kHz})$ bursts were generated by Tucker-Davis Technologies (TDT) System II hardware, driven by TDT Auditory Evoked Potential software, transduced by a Kef T27 speaker placed $0.65 \mathrm{~m}$ from the intact ear along the interaural axis, and calibrated with a Brüel and Kjær 0.5 inch microphone and measuring amplifier. Five-frequency audiograms were obtained from threshold assessments of the amplified and averaged ABRs before and after insertion of a plug in the intact ear. A previous behavioral determination of the effect of plugging showed an attenuation of $45-50 \mathrm{~dB}$ at $500 \mathrm{~Hz}$ (Hine et al., 1994).

After plug removal in the behavioral animals, the status of the left (previously plugged) ear was assessed in three ways before post-plug testing was started. The ear canal and tympanic membrane were inspected using conventional and fiber optic otoscopy. Tympanometry was performed using a Kamplex tympanometer that had been specially adapted for animal research. Finally, in four previously ear-plugged animals, five-frequency ABR audiometry was conducted on the left ear, as above, with a fresh earplug inserted in the right (previously unplugged) ear. Those data were compared with a sample of ABR audiograms from 10 normally hearing ferrets (not used in this study). Additional otoscopy and tympanometry were routinely performed when an earplug had been lost or during an occasional infection of the plugged ear. In general, the earplugs stayed in place well, except in some of the very young, preweanling infants. There was little persistent problem with infection, but two of the infant plugged animals had to be treated with antibiotics and have their earplug removed for a few days during the plug rearing period. Immediately after plug removal, three of the ferrets had varying amounts of cerumen in the ear canal. This was treated with veterinary ear wax softeners and, in two cases, with syringing. The success of the treatment was assessed with otoscopy, tympanometry, and on one occasion, audiometry, as described above. In the two cases of occlusion, the ear canal became patent within a few days of unplugging and was completely clear within 1-2 weeks. In every case, the ear was clean before post-plugging testing commenced.

Behavioral apparatus. Training and testing took place in a semicircular cage with a radius of $0.75 \mathrm{~m}$ (Fig. 1) (Hine et al., 1994), located inside a sound- and echo-attenuating chamber. A start area consisted of a metal plate on a raised platform, a wire mesh barrier, and a solenoid-operated water spout (spout 1). Contact between spout 1 and the metal plate completed the circuit for delivery of water from the spout and, for trial 
Figure 2. Performance of individual ferrets with varying tone level. Control and Bilateral condition (see Fig. 1) maskedthreshold data were obtained from three groups of animals: Normal (normally hearing adults), Infant-plug (plug inserted in the left ear in infancy, tested as adults), and Adult-plug (plug inserted in the left ear in adulthood). For the two "plug" groups, the data shown here were obtained with the plug inserted. In the top panels, actual psychometric functions show the mean percent correct discrimination (tone/no-tone) for each animal, averaged over two threshold determinations ("runs"). These functions were used to derive thresholds in the Final value method (see Testing in Materials and Methods). In the bottom panels, functions derived using the Logistic method are shown. One infant-plug ferret (9405, arrows) did not achieve the normal training criterion of $\geq 90 \%$.

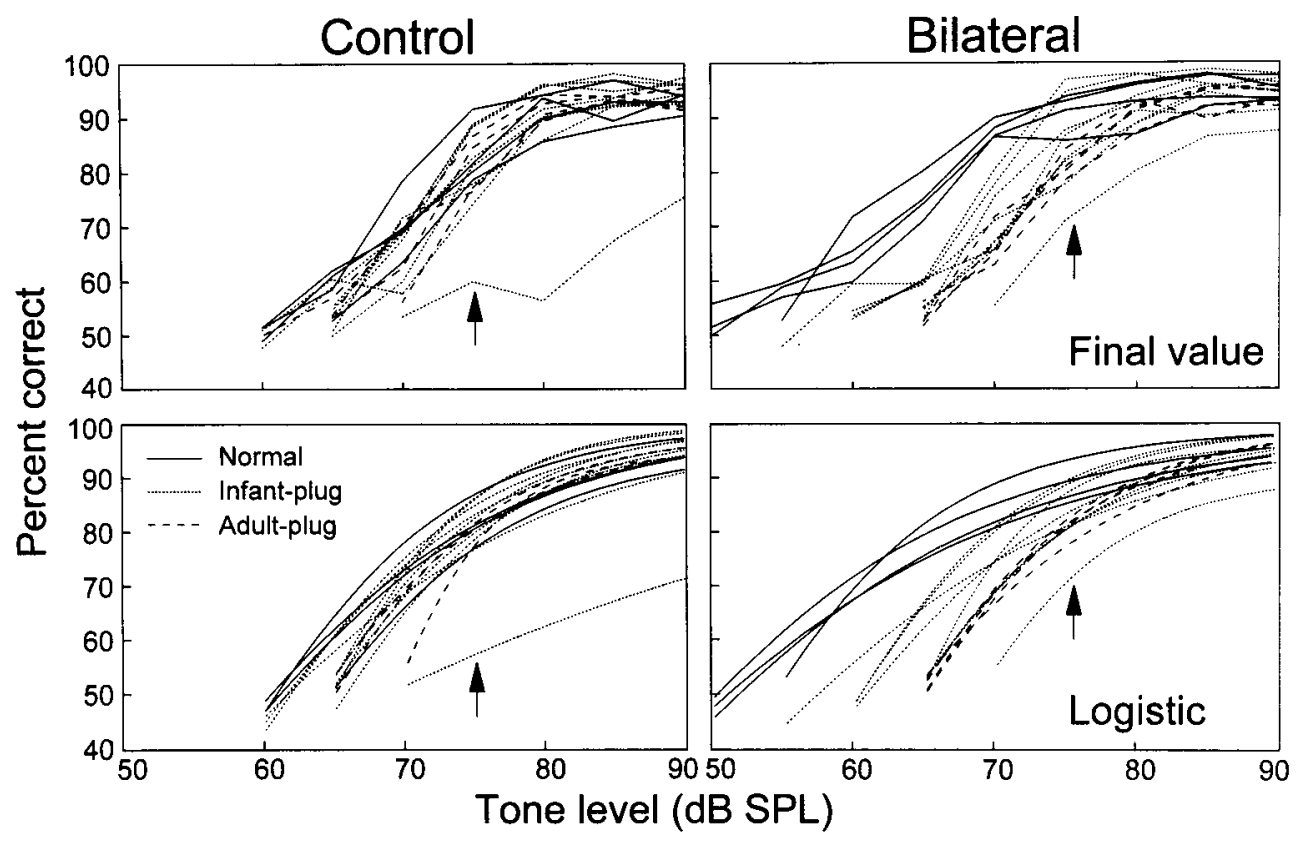

initiation, the brief illumination of a light $(10 \mathrm{~W})$ directly in front of the start area on the perimeter of the apparatus. Auditory stimuli were presented from two $10 \mathrm{~cm}$ midrange loudspeakers (R.S. Components), also on the perimeter and positioned as shown in Figure 1. Two additional water spouts were located $60^{\circ}$ right (spout 2) and $60^{\circ}$ left (spout 3 ) of the midline. Contact with metal plates on the chamber floor in front of each spout provided the circuit necessary for delivery of reinforcement. Sound generation. Signals were $500 \mathrm{msec}$ bursts (10 $\mathrm{msec}$ rise/fall times) of a $500 \mathrm{~Hz}$ pure tone, generated using an analog system and calibrated using spectral analysis (Cambridge Electronic Design "Waterfall") and a Brüel and Kjær microphone and measuring amplifier. Within the sound field around spout 1 , the SPL $\left(2 \times 10^{-5} \mathrm{~N} / \mathrm{m}^{2}\right)$ of the tone did not vary by more than $0.5 \mathrm{~dB}$. The maximum output of the delivery system was 90 dB SPL (rms, $\left.2 \times 10^{-5} \mathrm{~N} / \mathrm{m}^{2}\right)$. A single source of continuous band pass noise (center frequency $500 \mathrm{~Hz}, 120 \mathrm{~Hz}$ bandwidth at $3 \mathrm{~dB}$ down, 160 $\mathrm{dB} /$ octave filter skirts) was directed to two power amplifiers. One amplifier mixed the tone and noise, which were led to speaker A (Fig. 1). The second amplifier received noise alone, and its output was led to speaker B. The SPL and the phase of the noise from the two speakers were matched. For both the control and bilateral test conditions (Fig. 1), the level of the noise at spout 1 was $71 \mathrm{~dB}$ SPL $(50 \mathrm{~dB} / \mathrm{Hz}$ average spectrum level).

Training. Ferrets were trained to stand on the start platform, put their heads through the hole in the barrier, and initiate a trial by licking spout 1. Performance on this task was trained to a variable ratio (1:8) schedule. Trials on which a reward was presented at spout 1 were not recorded because of the noise made by the spout solenoid. Initially, contact with spout 1 triggered the presentation of either a "no-tone" trial, in which the trial onset light alone was activated, or a "tone" trial, in which a tone (70 $\mathrm{dB}$ SPL) was presented from speaker A along with the light. The probability of each type of trial was 0.5 . Ferrets were trained to go right (to spout 2 ) on a signal trial and left (to spout 3 ) on a no-signal trial. Incorrect responses were not rewarded, and those trials were repeated but not included in the estimate of performance. There were two 20- to 40-min sessions daily, $7 \mathrm{~d}$ a week. Each session had 10 practice trials, a block of 100 trials from which performance was calculated, and sufficient extra trials to satiate the animal. When the ferrets reached a criterion of $\geq 90 \%$ correct responses in three consecutive sessions, the continuous noise was introduced on speakers $\mathrm{A}$ and $\mathrm{B}$, set in the control configuration (Fig. 1), and the tone level was increased to $90 \mathrm{~dB}$ SPL. The new task was to discriminate between a tone-in-noise stimulus and a noise-alone stimulus, analogous to the previous tone, no-tone task. Again, the ferrets had to obtain $\geq 90 \%$ correct responses in three consecutive sessions to reach the training criterion. Note that one of the infant-plug ferrets (9405) failed to achieve this criterion (see Fig. 2).

Testing. After training, the level of the tone was reduced by $5 \mathrm{~dB}$ each time two consecutive test sessions produced statistically comparable $(p \leq 0.1)$ performance. This was continued until performance averaged over the last two sessions fell below $56.5 \%$ correct, the critical value for above-chance performance in 200 trials at the 0.05 level of significance. Two estimates of threshold were obtained. In the "final value" method, threshold was defined as that SPL for which performance was equal to $56.5 \%$ correct and was determined by linear interpolation between the two tone levels on either side of this point. Because this estimate depended entirely on the values of the final two tone levels, we chose a second method that used more of the available data set. In the "logistic" method, a three-parameter logistic (sigmoidal) function (SigmaPlot for Windows R4.0, SPSS) was fitted to the data obtained at each tone level:

$$
y=\frac{a}{1+\left(\frac{x}{x_{\mathrm{o}}}\right)^{\mathrm{b}}} .
$$

Threshold was defined as the tone level at which the fitted function intersected the $56.5 \%$ correct performance level (see Fig. 2).

After threshold determination with the speakers in the control configuration, $90^{\circ}$ to the ferret's right (Fig. 1), speaker B was moved to $90^{\circ}$ to the ferret's left (Fig. 1, Bilateral). The training criterion at a tone level of $90 \mathrm{~dB}$ was reestablished, and testing was conducted as before. The only difference between the control and bilateral configurations was the position of speaker B.

The complete control and bilateral test conditions were repeated twice in each group of animals, with the bilateral tests preceding the control tests the second time around. After removal of the plug in the earplug groups, tests recommenced within $0-4$ weeks, and further tests were performed during variable periods of up to 22 months as detailed above and in Results.

Performance levels obtained from psychophysical experiments requiring binary responses to single-interval trials, such as those used here, may be affected by response biases. However, it has previously been established in this task that measuring performance as a percentage of correct responses gives a true estimate of the ferrets' sensory capability that is not affected by systematic variation in response biases (Hine et al., 1994).

\section{RESULTS}

Most of the ferrets learned readily to detect the tone in the control condition and, at the highest tone level (90 dB SPL), generally reached the training criterion of consistent, $\geq 90 \%$ correct responses within approximately 20-30 sessions, as reported previously (Hine et al., 1994). Figure 2 shows the individual psychometric functions for all the animals in each test condition, averaged from two threshold determinations, and obtained, 


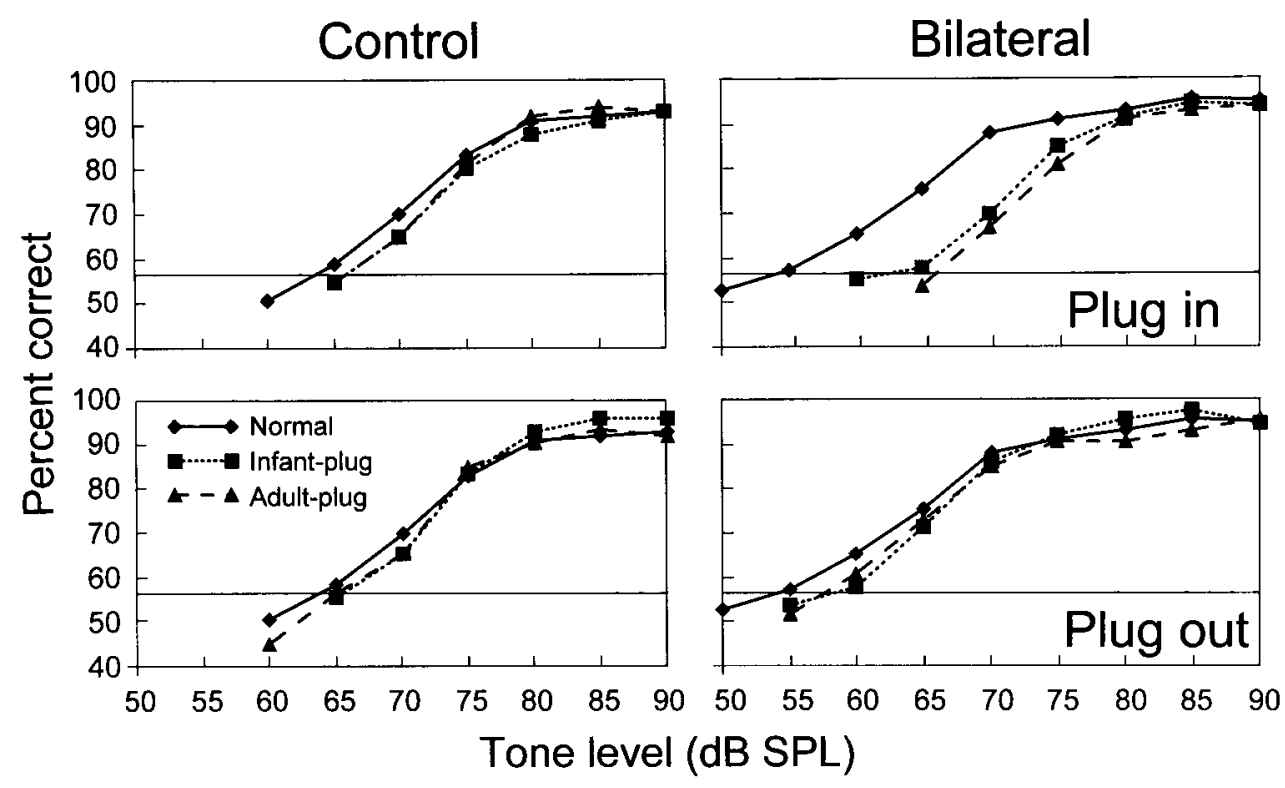

Figure 3. Mean performance of ferret groups. "Final value" masked-threshold psychometric functions from individuals (see Fig. 2) have been averaged and are shown for the two plug groups with the plug inserted (top panels) and in the first threshold determinations after plug removal (bottom panels). Data for the normal ferrets (without plugs) are reproduced in both sets of panels. Threshold levels $(56.5 \%$ correct; see Testing in Materials and Methods) are indicated by the horizontal lines. Ear plugging reduced $B i$ lateral thresholds without substantially changing Control thresholds. where appropriate, when the ear plugs were inserted. Figure 3 (top panels) shows the same normal and "plug in" data averaged between the individuals within each group. When the tone level was decreased, performance began to decline at a rate that was determined by the test condition and by the history of ear plugging. For the normal ferrets, performance in both the control and bilateral conditions remained high until the tone level was decreased below $80 \mathrm{~dB}$ SPL (Figs. 2, 3). Performance then started to decline, but it did so much more rapidly in the control than in the bilateral condition. The superior performance of these animals in the bilateral condition was therefore enhanced at lower tone levels. Little difference was found between the thresholds (at $56.5 \%$ correct) for either condition produced by the logistic and final value methods (absolute mean difference $=0.51 \mathrm{~dB}$ for all data) (Fig. 2). Thus, the simpler, final value method was used for the remainder of the analysis. The mean tone threshold difference between the bilateral and control conditions (the mean BU) for the normal ferrets was $10.1 \mathrm{~dB}$ (Fig. 4).

\section{Effect of ear plugging}

We showed previously (Hine et al., 1994) that plugging the left ear of adult ferrets just before testing led to markedly reduced performance in the bilateral condition and a slight reduction in performance in the control condition. Together, these threshold shifts abolished BU. Longer term (6-8 months) plugging in the adult-plug group also abolished BU by selectively increasing the masked threshold in the bilateral condition (Figs. 2-5). In this group, there was no sign of adaptation to the plug. In the infantplug group, tests of BU while the ear was plugged were conducted between 6 and 10 months of age. Within this range, no systematic differences were noted in masked thresholds as a function of age or test experience (data not shown). The results from members of both plug groups were therefore averaged into single values for each animal (Fig. 5, Plug in data). With the ear plugged, one infant-plug ferret (9440) returned a BU of $7.7 \mathrm{~dB}$, but the mean of the infant-plug group (2.3 dB) (Fig. 4) was not significantly higher than that of the adult-plug group $\left(0.7 \mathrm{~dB} ; t_{10}=0.81\right)$, again providing no clear sign of adaptation to the plug. Ferret 9405, the infant-plug animal that failed to achieve the training criterion (Fig. 2), and that had the right ear plugged, had $2.0 \mathrm{~dB}$ of BU and

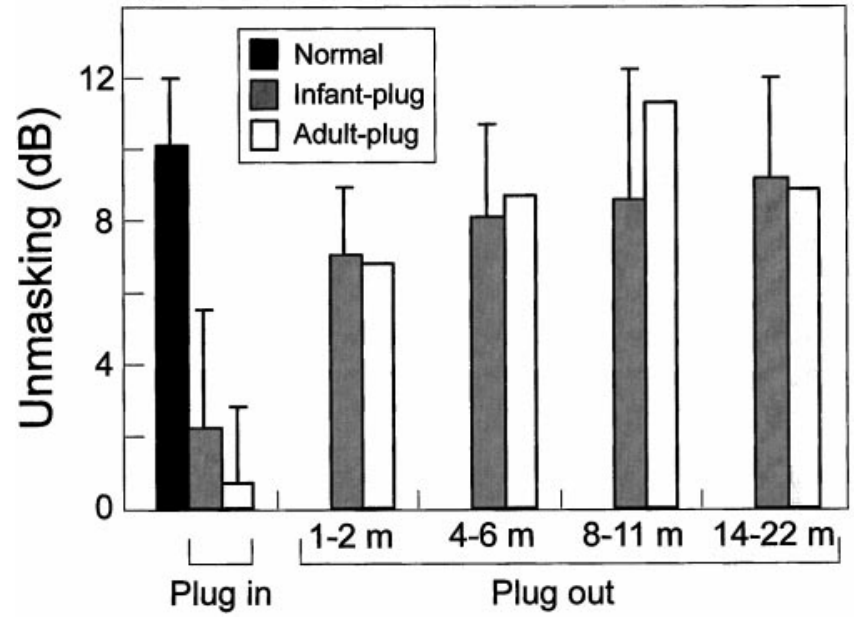

Figure 4. Binaural unmasking. For each plug group, the mean (and the $\mathrm{SD})$ level of unmasking is shown during plugging (Plug in) and at various times [in months $(m)$ ] after unplugging (Plug out). BU for each animal was calculated by subtracting the bilateral threshold from the control threshold. The number of animals contributing to each histogram bar can be found by reference to Figure 5. Error bars are not provided for the Adult-plug group after plug removal because only two animals were tested 1-11 $\mathrm{m}$ after unplugging and only one animal was tested at $14-22 \mathrm{~m}$. BU was abolished throughout long-term ear plugging and transiently reduced, relative to normal, after plug removal.

therefore did not markedly differ from or affect the mean of the whole group.

\section{Plug removal}

For both the infant- and adult-plug groups, removal of the plug led to a partial restoration of BU within 1-2 months (Fig. 4). This initial restoration was wholly due to improved sensitivity to the tone in the bilateral condition; thresholds in the control condition were the same as those recorded before plugging (Fig. 5). In those cases where thresholds were determined at shorter delays after plug removal ( 2 weeks was the minimum), similar masked thresholds were obtained. However, the level of BU in the first post-plug test period for which group data were compiled (1-2 months) was significantly less than that obtained in the normal animals that 
Figure 5. Masked thresholds. The individual animal masked thresholds from which the data in Figure 4 were derived are shown separately for the Control and Bilateral conditions (see Fig. 1). $N$, Normal group; $I$, Infant-plug group; $A$, Adultplug group.

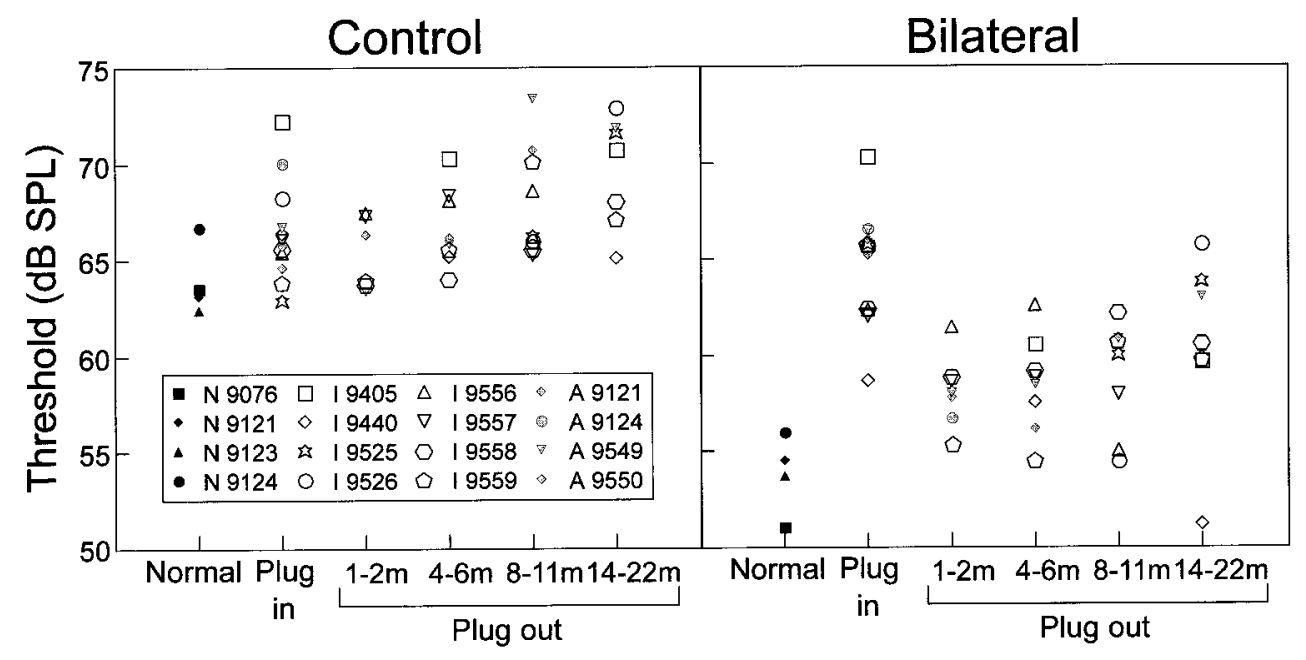

had never experienced an ear plug $\left(t_{8}=2.7, p=0.03\right.$, two-tailed). As time progressed after unplugging, BU gradually increased (Fig. 4). In the second test period (4-6 months), the difference between the formerly plugged and the normal animals was no longer significant $\left(t_{10}=1.5, p=0.18\right)$. Further increases in BU in the $8-11$ and 14-22 month post-plug groups led to normal levels of BU. No differences were found between the infant- and adultplug groups (Fig. 5), either in the initial thresholds or in the subsequent recovery. However, only two adult-plug ferrets were examined beyond the first post-plug test period. To increase statistical power, all plugged animals were therefore included in the recovery analyses reported above.

\section{Status of plugged ear}

After removal of the ear plug, and treatment with ear cleaning fluid where necessary, all of the previously plugged ear canals attained a clean and unimpeded appearance (see Materials and Methods for further details). Tympanometry produced type A tympanogams (Shanks et al., 1988), indicating a clear passage through to and normal compliance of the tympanic membrane. Where ABR audiograms were obtained (Fig. 6), thresholds were generally within the range measured in a large sample of normal ferret ears that had never been plugged. Note, however, that a small, progressive insensitivity of hearing occurred in both the control and bilateral masked thresholds of several of the formerly plugged ferrets in the months after plug removal (Fig. 5). This is suggestive of an age-related hearing impairment because (1) the control thresholds were normal soon after unplugging (1-2 months), and (2) the very long survivals (to an absolute age of 31 months, the oldest animals tested) represented the only circumstance under which the control thresholds were found to be elevated. It is possible that an age-related impairment accounted for the elevated ABR threshold of ferret 9557 at $1 \mathrm{kHz}$ (Fig. 6).

\section{Attenuating effect of plug}

The plug produced a relatively flat hearing loss of $\sim 40 \mathrm{~dB}$ across the range $1-16 \mathrm{kHz}$, as measured by ABR audiometry (Fig. 7). Additional behavioral measurements (Hine et al., 1994) suggested a similar or slightly higher level of attenuation at $500 \mathrm{~Hz}$. The latter measurements [and others by Kelly et al. (1986)] also showed that the absolute threshold of the ferret at $500 \mathrm{~Hz}$ is $\sim 25$ $\mathrm{dB}$ SPL. Thus, at the higher levels used ( $\geq 80 \mathrm{~dB}$ SPL), the tone should have been clearly audible, in both ears and in both conditions, with the plug inserted. At lower levels, the tone would soon

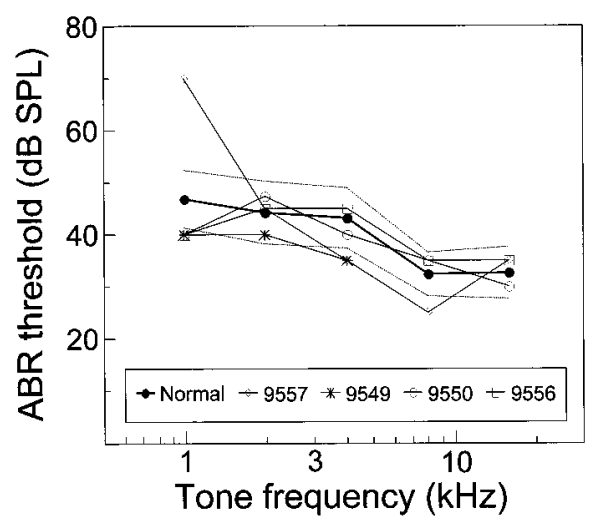

Figure 6. Auditory brainstem response $(A B R)$ audiograms. The mean audiogram for 10 normal ferrets is shown as a bold line connecting filled circles. The unmarked, dotted lines on either side of this show the $95 \%$ confidence intervals of the normal means. Data are also shown for four ear-plugged ferrets, measured when the left (normally/previously plugged) ear was open and the right ear was plugged. For two of these ferrets (adult-plug, 9549, 9550) data were obtained early during the plug-in period. For the remaining ferrets (infant-plug, 9556, 9557) data were obtained 11 months after unplugging.

become inaudible in the plugged ear. Because the interaural level difference (ILD) in the ferret for $300-700 \mathrm{~Hz}$ tones presented $90^{\circ}$ to the midline is negligible (Carlile, 1990), we may assume that in both stimulus conditions the masking noise would be marginally audible in the plugged ear, because its constant level (71 dB SPL) approximated the hearing level predicted from the sum of the plug attenuation and the audiometric threshold. This analysis suggests that at low tone levels with the plug inserted, the control and bilateral conditions would produce very nearly identical acoustic input to each ear, presumably accounting for the closely similar performance of the plugged animals in the two conditions.

\section{DISCUSSION}

The main finding of this study was that long-term unilateral conductive hearing loss, either in infancy or in adulthood, produced a long-lasting, but transient, deficit in binaural hearing after the restoration of normal peripheral function. This result is consistent with reports that BU in humans is transiently depressed after OME in children (Moore et al., 1991; Pillsbury et al., 1991; Hall et al., 1995b; Hogan et al., 1996) and otosclerosis in adults (Hall et al., 1990). These results suggest that the central 


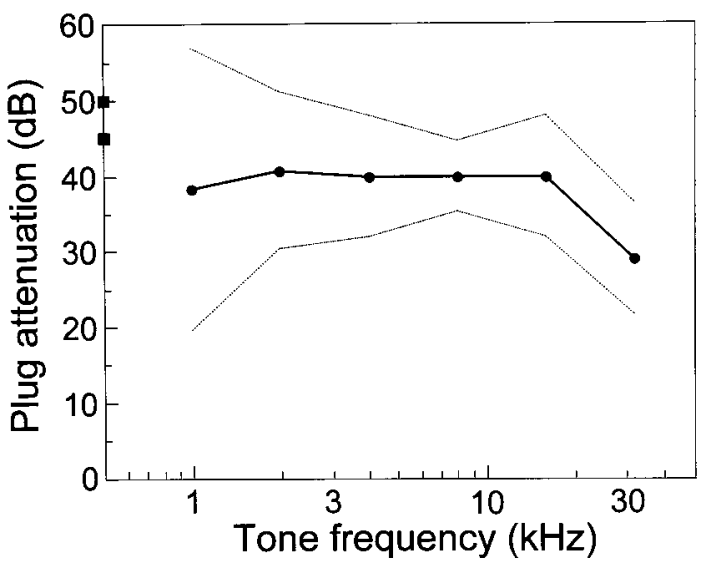

Figure 7. Attenuating effect of ear plugs. Mean data $(\bullet, \pm 95 \%$ confidence intervals) from three adult ferrets. The ferrets were deafened in one ear and had ABR audiograms recorded in the other ear before and after insertion of an ear plug that was identical to those used in the trained ferrets. The behaviorally determined threshold (Hine et al., 1994) of an additional two ferrets to a $500 \mathrm{~Hz}$ tone is indicated by the filled squares on the ordinate.

auditory system is dynamically influenced by auditory experience, presumably analogous to other demonstrations of long-term adaptation and training in the auditory system (Recanzone et al., 1993) and elsewhere (Gilbert, 1998).

There is, however, an alternative interpretation. Infant-plug ferrets did not receive normal binaural experience until they were at least 8 months old. During this time, their ability to perform $\mathrm{BU}$ and, presumably, other binaural tasks was greatly impaired or abolished. Yet within 2-4 weeks of their first experience of normal hearing, the ferrets showed substantial BU. Within another 3-4 months, BU was normal. Thus, there was little or no adaptation to the ear plug, and a relatively rapid recovery occurred after the onset of normal experience. These observations suggest a central auditory system that is essentially hard-wired and insensitive even to major input perturbations.

Which of these interpretations is correct? To answer this question it is necessary to consider further the nature of conductive hearing losses, the mechanisms of $\mathrm{BU}$, and the place of the present observations among other reports of auditory system plasticity.

\section{Conductive hearing losses}

The ear plugs used in this study produced an attenuation of 30-50 $\mathrm{dB}$. This is similar to the attenuation produced by other ear plugs (Knudsen et al., 1984a; McPartland et al., 1997), other forms of ear canal occlusion (Moore and Irvine, 1981; Wilmington et al., 1994), and manipulations and diseases of the middle ear (Tucci and Rubel, 1985; Hall et al., 1990; Bluestone and Klein, 1995). In addition to reducing air-conducted sound levels, these treatments and conditions can potentiate low-frequency, bone-conducted sounds accompanying self-vocalizations (Tonndorf, 1972). Both direct (Knudsen et al., 1984a) and indirect (Hall et al., 1995a; Hogan et al., 1995) measurements suggest that ear occlusions can also alter the temporal properties of acoustic signals passing to the inner ear. Thus, the acoustic effects of ear occlusions are complicated.

Variations in the acoustic and peripheral pathological effects of conductive losses may account for the sometimes confusing and inconsistent reports of their central consequences. Several studies have examined the effects of monaural occlusion during development on neural responses to ILD in mammals (Silverman and Clopton, 1977; Moore and Irvine, 1981; Brugge et al., 1985) and barn owls (Mogdans and Knudsen, 1992, 1994). The mammalian results have been inconsistent, but the barn owl results have shown systematic shifts in neural ILD tuning. The effects of monaural occlusion during infancy on the organization of auditory "space maps" in the superior colliculus (SC) have also been examined in owls (Knudsen, 1985) and ferrets (King et al., 1988). In both cases, compensatory shifts in the auditory spatial tuning of SC neurons have been reported. However, bilateral removal of the external ear structures alters the responses of SC neurons in different ways in these two species (Knudsen et al., 1994a,b; Schnupp et al., 1998).

Behavioral studies in barn owls have shown that adaptation to a conductive hearing loss occurs only after deprivation early in life, whereas recovery from an early deprivation can occur much later, but not after sexual maturity. In both of these respects, the present results in the ferret differed from those in the barn owl.

There were two obvious differences between our experimental methods and those used in the barn owl. The first was the species studied. Barn owls can localize accurately only sounds having frequency components in the $4-9 \mathrm{kHz}$ range but, in contrast to mammals, can use both interaural time differences (ITDs) and ILDs together to localize sounds within this frequency range (Konishi, 1993). Moreover, although the owl's external ear generates prominent spectral localization cues (Keller et al., 1998), sound localization in this species is generally thought to rely solely on ITDs and ILDs.

The second difference was the nature of the task. In the barn owls, free-field localization was used to assess adaptation, whereas the present study used BU. It is unknown whether owls have BU or whether they could use BU in the same way as mammals, although recent experiments in budgerigars (Dent et al., 1997) have shown that birds can have BU. Wilmington and colleagues (1994) compared directly the effect of congenital ear canal atresia on free-field localization and BU in humans. They showed more extensive and longer lasting effects of the atresia on localization than on $\mathrm{BU}$, although poor performance on both tasks was found immediately after surgical correction of the atresia. Our own preliminary data on ferrets reared with ear plugs also show considerable adaptation in free-field localization during plug wearing (Parsons et al., 1999), as did a recent adult human study using outer ear inserts that altered spectral cues (Hofman et al., 1998). These experiments thus show highly task-dependent types and degrees of adaptation to conductive perturbations of hearing. In general, there appears to be a greater adaptive influence of auditory experience on localization than on other binaural tasks.

\section{Occlusion and binaural unmasking}

Occlusion of one ear could reduce BU in several ways. In normally hearing human listeners, reducing sound level to one ear reduces BU (Colburn and Durlach, 1965), and the overall noise level is directly correlated with BU (Durlach and Colburn, 1978). As the level of sound in the occluded ear approaches the threshold for hearing, the task translates into one of monaural masking. At higher sound levels, if the plug produces a small delay in the signal passing around and through it, this may result in both a change in the interaural phase of the tone and noise and a spectral complexity attributable to the effect of a "delay and add" mechanism. Finally, as the level of airborne sound reaching the 
occluded ear is greatly reduced, bone conduction pathways, transmitting low-pass-filtered sounds, may provide significant input (Tonndorf, 1972). It is unclear which of these mechanisms contributes to the impaired BU found after plug removal. However, because the ILD produced by the plug is large, and because ILDs make a major contribution to free-field $\mathrm{BU}$, at least in humans (Bronkhorst and Plomp, 1988), it may be that an adaptation to the ILD produced by the plug results in poor unmasking after unplugging. Certain behavioral (Florentine, 1976; Parsons et al., 1999) and physiological (Silverman and Clopton, 1977; Moore and Irvine, 1981; King et al., 1988) data support this interpretation, but others (Brugge et al., 1985; McPartland et al., 1997) have failed to find clear adaptation to altered binaural cues in mammals.

\section{Auditory system dynamics}

In this study, prolonged unilateral ear plugging produced a transient change in the way the auditory system processed binaural stimuli. This occurred in both infant- and adult-plugged ferrets. Similar results have been obtained in children and adult humans after prolonged conductive hearing loss (see introductory remarks). Here we discuss the possibility that such reversible changes in binaural processing are produced by neural mechanisms analogous or identical to those demonstrated in auditory perceptual training (Recanzone et al., 1993; Kilgard and Merzenich, 1998a,b; Weinberger and Bakin, 1998). A fundamental aspect of that training is the repetitive, associative pairing of particular auditory cues with reinforcement. For example, in asymmetric conductive hearing loss, normally inappropriate binaural cues are repetitively paired with other cues (e.g., visual), presumably leading to a conditioned association between the inappropriate cues and various reinforcers, such as conspecific vocalizations or the water spouts used here. This association can develop more (Recanzone et al., 1993; Kilgard and Merzenich, 1998a, b) or less (Weinberger and Bakin, 1998) gradually and is manifested as a long-lasting change in both the perception of the reinforced cues and the physiology of auditory cortical neurons. In the BU task, we found little evidence for learning of (i.e., adaptation to) the inappropriate cues provided by the earplug. However, we did show a change in perception of normal cues that was long lasting. This altered perception was gradually changed (normalized) by a new set of (appropriate) cues. Interestingly, this second change occurred over a time frame (weeks) that was similar to several other instances of auditory "learning" in adults (Robertson and Irvine, 1989; Recanzone et al., 1993; Gilbert, 1998; Hofman et al., 1998).

The reversible nature of the binaural impairment found after unilateral earplugging suggests that OME and other clinical forms of conductive hearing loss may lead to a form of auditory neural learning. Recent data indicate that at least one aspect of binaural discrimination (ILD) can also be improved in normally hearing individuals through additional training (Wright and Fitzgerald, 1999). These data, together with the clinically demonstrated reversal of binaural impairments after recovery of normal binaural sound transmission (Wilmington et al., 1994; Hall et al., 1995; Hogan and Moore, 1996), support the neural learning hypothesis. However, the failure to adapt to the plug (while inserted) suggests limits to this learning and may be indicative of a binaural system that is essentially hard-wired. Auditory experience and training may thus contribute to relatively subtle recalibrations of the system, such as learning to use the altered spectral cues produced by an ear plug or insert, or (re)learning to take advantage of normal binaural input.

\section{REFERENCES}

Bluestone CD, Klein JO (1995) Otitis media in infants and children, Ed 2. Philadelphia: W. B. Saunders.

Bronkhorst AW, Plomp R (1988) The effect of head-induced interaural time and level differences on speech intelligibility in noise. J Acoust Soc Am 83:1508-1516.

Brugge JF, Orman SS, Coleman JR, Chan JCK, Phillips DP (1985) Binaural interactions in cortical area AI of cats reared with unilateral atresia of the external ear canal. Hear Res 20:275-287.

Carlile S (1990) The auditory periphery of the ferret. I: Directional response properties and the pattern of interaural level differences. J Acoust Soc Am 88:2180-2195.

Colburn HS, Durlach NI (1965) Time-intensity relations in binaural unmasking. J Acoust Soc Am 38:93-103.

Dent ML, Larsen, ON, Dooling RJ (1997) Free-field binaural unmasking in budgerigars (Melopsittacus undulatus). Behav Neurosci 111:590-598.

Durlach NI, Colburn HS (1978) Binaural phenomena. In: Handbook of perception, Vol IV, Hearing (Carterette EC, Friedman MD, eds), pp 365-466. New York: Academic.

Feng AS, Rogowski BA (1980) Effects of monaural and binaural occlusion on the morphology of neurons in the medial superior olivary nucleus of the rat. Brain Res 189:530-534.

Florentine M (1976) Relation between lateralization and loudness in asymmetrical hearing losses. J Am Audiol Soc 1:243-251.

Gilbert CD (1998) Adult cortical dynamics. Physiol Rev 78:467-485.

Haggard MP, Hughes E (1993) Objectives, values and methods of screening children's hearing: a review of the literature. London: HMSO.

Hall JW, Grose JH, Pillsbury HC (1990) Predicting binaural hearing after stapedectomy from presurgery results. Arch Otolaryngol Head Neck Surg 116:946-950.

Hall JW, Grose JH, Mendoza LL (1995a) Masker interaural phase and the MLD: effects of conductive hearing loss. Hear Res 84:91-98.

Hall JW, Grose JH, Pillsbury HC (1995b) Long-term effects of chronic otitis media on binaural hearing in children. Arch Otolaryngol Head Neck Surg 121:847-852.

Hine JE, Martin RL, Moore DR (1994) Free-field binaural unmasking in ferrets. Behav Neurosci 108:196-205.

Hofman PM, Van Riswick JGA, Van Opstal AJ (1998) Relearning sound localization with two ears. Nat Neurosci 1:417-421.

Hogan SC, Pralong D, Moore DR (1995) Effects of unilateral earplugging in humans on binaural unmasking. Br J Audiol 29:56-57.

Hogan SC, Meyer SE, Moore DR (1996) Binaural unmasking returns to normal in teenagers who had otitis media in infancy. Audiol Neurol Otol 1:104-111.

Hogan SC, Stratford KJ, Moore DR (1997) Duration and recurrence of otitis media with eff usion in children from birth to 3 years: prospective study using monthly otoscopy and tympanometry. $\mathrm{Br}$ Med J 314:350-353

Keller CH, Hartung K, Takahashi TT (1998) Head-related transfer functions of the barn owl: measurement and neural responses. Hear Res 118:13-34.

Kelly JB, Kavanagh GL, Dalton JCH (1986) Hearing in the ferret (Mustela putorius): thresholds for pure tone detection. Hear Res 24:269-275.

Kilgard MP, Merzenich MM (1998a) Cortical map reorganization enabled by nucleus basalis activity. Science $279: 1714-1718$.

Kilgard MP, Merzenich MM (1998b) Plasticity of temporal information processing in the primary auditory cortex. Nat Neurosci 1:727-731.

King AJ, Hutchings ME, Moore DR, Blakemore C (1988) Developmental plasticity in the visual and auditory representations in the mammalian superior colliculus. Nature 332:73-76.

Knudsen EI (1985) Experience alters the spatial tuning of auditory units in the optic tectum during a sensitive period in the barn owl. J Neurosci 5:3094-3109.

Knudsen EI, Esterly SD, Knudsen PF (1984a) Monaural occlusion alters sound localization during a sensitive period in the barn owl. J Neurosci 4:1001-1011.

Knudsen EI, Knudsen PF, Esterly SD (1984b) A critical period for the recovery of sound localization accuracy following monaural occlusion in the barn owl. J Neurosci 4:1012-1020.

Knudsen EI, Esterly SD, Olsen JF (1994) Adaptive plasticity of the 
auditory space map in the optic tectum of adult and baby barn owls in response to external ear modification. J Neurophysiol 71:79-94.

Konishi M (1993) Listening with two ears. Sci Am 268:66-73.

Lucente FE, Sobol SM (1988) Essentials of otolaryngology, Ed 2. New York: Raven.

McPartland JL, Culling JF, Moore DR (1997) Changes in lateralization and loudness judgements during one week of unilateral ear plugging. Hear Res 113:165-173.

Mogdans J, Knudsen EI (1992) Adaptive adjustment of unit tuning to sound localization cues in response to monaural occlusion in developing owl optic tectum. J Neurosci 12:3473-3484.

Mogdans J, Knudsen EI (1994) Site of auditory plasticity in the brain stem (VLVp) of the owl revealed by early monaural occlusion. J Neurophysiol 72:2875-2891.

Moore BCJ (1997) An introduction to the psychology of hearing, Ed 4. San Diego: Academic.

Moore DR (1993) Plasticity of binaural hearing and some possible mechanisms following late-onset deprivation. J Am Acad Audiol 4:277-283.

Moore DR, Hine JE (1992) Rapid development of auditory brainstem response thresholds in individual ferrets. Dev Brain Res 66:229-235.

Moore DR, Irvine DRF (1981) Plasticity of binaural interaction in the cat inferior colliculus. Brain Res 208:198-202.

Moore DR, Kowalchuk NE (1988) Auditory brainstem of the ferret: effects of unilateral cochlear lesions on cochlear nucleus volume and projections to the inferior colliculus. J Comp Neurol 272:503-515.

Moore DR, Hutchings ME, King AJ, Kowalchuk NE (1989) Auditory brainstem of the ferret: some effects of rearing with a unilateral ear plug on the cochlea, cochlear nucleus, and projections to the inferior colliculus. J Neurosci 9:1213-1222.

Moore DR, Hutchings ME, Meyer SE (1991) Binaural masking level differences in children with a history of otitis media. Audiology 30:91-101.

Morey AL, Carlile S (1990) Auditory brainstem of the ferret: maturation of the brainstem auditory evoked response. Dev Brain Res 52:279-288.

Parsons CH, Lanyon RG, Moore DR, King AJ (1999) Auditory localization in the ferret: adaptation to chronic monaural occlusion during infancy. Assoc Res Otolaryngol Abstr 22:136.
Pillsbury HC, Grose JH, Hall JW (1991) Otitis media with effusion in children: binaural hearing before and after corrective surgery. Arch Otolaryngol Head Neck Surg 117:718-723.

Recanzone GH, Schreiner CE, Merzenich MM (1993) Plasticity in the frequency representation of primary auditory cortex following discrimination training in adult owl monkeys. J Neurosci 13:87-103.

Robertson D, Irvine DRF (1989) Plasticity of frequency organization in auditory cortex of guinea pigs with partial unilateral deafness. J Comp Neurol 282:456-471.

Schnupp JWH, King AJ, Carlile S (1998) Altered spectral localization cues disrupt the development of the auditory space map in the superior colliculus of the ferret. J Neurophysiol 79:1053-1069.

Shanks JE, Lilly DJ, Margolis RH, Wiley TL, Wilson RH (1988) Tympanometry. J Speech Hear Disord 53:354-377.

Silverman MS, Clopton BM (1977) Plasticity of binaural interaction. I. Effect of early auditory deprivation. J Neurophysiol 40:1266-1274.

Slattery 3rd WH, Middlebrooks JC (1994) Monaural sound localization: acute versus chronic unilateral impairment. Hear Res 75:38-46.

Smith ZD, Gray L, Rubel EW (1983) Afferent influences on brainstem auditory nuclei of the chicken: $n$. laminaris dendritic length following monaural conductive hearing loss. J Comp Neurol 220:199-205.

Tonndorf J (1972) Bone conduction. In: Foundations of modern auditory theory, Vol 2 (Tobias JV, ed), pp 195-237. New York: Academic.

Tucci DL, Rubel EW (1985) Afferent influences on brain stem auditory nuclei of the chicken: effects of conductive and sensorineural hearing loss on n. magnocellularis. J Comp Neurol 238:371-381.

Weinberger NM, Bakin JS (1998) Learning-induced physiological memory in adult primary auditory cortex: receptive fields plasticity, model, and mechanisms. Audiol Neurootol 3:145-167.

Wiesel TN (1982) Postnatal development of the visual cortex and the influence of environment. Nature: 299:583-591.

Wilmington D, Gray L, Jahrsdoerfer R (1994) Binaural processing after corrected congenital unilateral conductive hearing loss. Hear Res 74:99-114.

Wright BA, Fitzgerald MB (1999) Learning and generalization of interaural-level-difference discrimination by humans. Assoc Res Otolaryngol Abstr 22:58. 